\title{
Catheter-Associated Venous Thromboembolism in Patients with Cystic Fibrosis
}

\author{
Kamyar Afshar*, Ali Ahoui, Debbie S Benitez and Purush Rao A \\ Division of Pulmonary and Critical Care Medicine, University of Southern California, Keck School of Medicine, USA
}

\begin{abstract}
Catheter-associated Venous Thromboembolism (VTE) is a common complication encountered in a tertiary care center. The purpose of this analysis was to evaluate the incidence of catheter-associated VTE phenomenon and assess for potential risk factors which may contribute to higher risks specifically in patients with cystic fibrosis. Overall implantable vascular access devices appear to be relatively safe and reliable for long term and frequent intravenous antibiotic therapy in patients with cystic fibrosis.
\end{abstract}

Keywords: Cystic fibrosis; Thromboembolus; Catheter-associated

\section{Introduction}

Cystic Fibrosis (CF) is a multi-system disease that results in the excessive production of thick secretions within most vital organs. All CF patients will have pulmonary involvement of the disease. Pulmonary complications in this population are ensued with the repeated episodes of mucus insippation and inflammation resulting in bronchiectasis, pneumonia, allergic bronchopulmonary aspergillosis, hemoptysis and pneumothorax. Pulmonary infectious exacerbations are the most common cause of hospitalization (48\%) in patients with cystic fibrosis [1]. Depending on the severity of lung function impairment and adherence to maintenance therapy, patients may have an annual exacerbation rate as little as none or up to four to five times per year. Management of CF patients experiencing a severe pulmonary exacerbation include initiation or increased aggressive airway clearance therapy, continued exercise regimen and inhalation therapies and the institution of intravenous anitmicrobial therapy of a an 10-14 day duration. Antibiotic selection is based on sensitivities of the most recent sputum isolates.

Maintaining patency of short-term peripheral IV catheters may be challenging due to the harsh effect of the antibiotics on the vessel, frequent occlusion and infiltration. Peripherally Inserted Central Catheters (PICC) and long-term indwelling catheters are proven alternatives to short term venous catheters for the administration of various medications. These techniques are utilized frequently in patients with cystic fibrosis to ease patient discomfort during prolonged duration of intravenous antimicrobial therapy for acute infectious exacerbations. Like other procedures, long-term catheters have complications that need to be discussed with patients. Currently available reports indicate a complication rate from 4.7 to $16 \%[2,3]$. We specifically sought out to evaluate the incidence of Venous Thromboembolic (VTE) phenomenon related to this procedure and assess for potential risk factors which may contribute to higher risks with specific patients with cystic fibrosis.

\section{Methods}

A retrospective study was performed of adults with CF who were hospitalized for pulmonary exacerbation during a 5-year period from 2005 through 2010 and had a long-term central venous catheter placement. Institutional review board approval was obtained and informed consent was waived. The USC Adult CF Clinic is a tertiary care center that treats 199 adults with CF. All patients with an indwelling catheter at the time of admission were identified. The average duration of a hospitalization for an exacerbation at the USC Center is 14 days.

Only peripherally inserted central catheters per admission and Port-A-Cath (Pharmacia Nu Tech; Piscataway, NJ) were included in this review. During the observed period, there were only 2 patients with Midline catheters (Landmark; Menlo Care, Menlo Park, CA). These patients were excluded as the catheters were not completely secured and had migrated out after 3 and 5 days respectively. The PICCs used during the time period were the Power-PICC 5-French polyurethane catheters (Bard Access System; Salt Lake City, UT). All PICCs were inserted under a sterile fashion by nurses or radiologists specialty trained under ultrasound guidance. The PICC line was inserted through the basilic vein in all cases. Chest radiographs were used to confirm adequate catheter tip positioning. The catheters were flushed with $10 \mathrm{cc}$ of normal saline followed by $3 \mathrm{cc}$ of heparin $(100 \mathrm{U} / \mathrm{cc})$ when not in use. Antibiotic infusions are over a 3-hour duration if a beta-lactam was ordered and over 1 hour if an aminoglycoside or quinolone was being administered.

Clinical suspicion for the development of a catheter associated VTE included signs and symptoms of extremity swelling or tenderness, erythema at or proximal to the insertion site, and difficulty in infusing or drawing from the catheter. Investigations to confirm the presence of thrombosis was performed by a vascular ultrasound. We do not routinely screen for asymptomatic patients for a catheter-associated VTE. A catheter-associated VTE was defined a thrombus adherent to the vein or surrounding the catheter. We reviewed medical records for identifying the number of catheter-associated VTEs and predisposing risk factors. Risk factors included smoking history, the use of contraceptives, presence of malignancy or nephropathy, coagulation profile (INR) and assessing the degree of inflammation (CRP). Comparisons between groups with the development of an associated VTE and those without were analyzed with chi square $\left(\mathrm{x}^{2}\right)$ calculation in categorical values and with independent $t$-test for numerical values.

\section{Results}

One hundred sixteen patients with cystic fibrosis were hospitalized over the 5 year period between 2005 and 2010. 62 patients were excluded due to not having a PICC or Port-a-Cath. Of the remaining

*Corresponding author: Kamyar Afshar, Division of Pulmonary and Critical Care Medicine, University of Southern California, Keck School of Medicine 2020 Zonal Avenue, IRD 723, Los Angeles, CA 90033, USA, Tel: 323-226-7923; Fax: 323-2262738; E-mail: kafshar@usc.edu

Received January 27, 2013; Accepted March 26, 2013; Published March 29 2013

Citation: Afshar K, Ahoui A, Benitez DS, Purush Rao A (2013) Catheter-Associated Venous Thromboembolism in Patients with Cystic Fibrosis. J Pulmon Resp Med 3: 143. doi:10.4172/2161-105X. 1000143

Copyright: @ 2013 Afshar K, et al. This is an open-access article distributed under the terms of the Creative Commons Attribution License, which permits unrestricted use, distribution, and reproduction in any medium, provided the original author and source are credited. 
Citation: Afshar K, Ahoui A, Benitez DS, Purush Rao A (2013) Catheter-Associated Venous Thromboembolism in Patients with Cystic Fibrosis. J Pulmon Resp Med 3: 143. doi:10.4172/2161-105X. 1000143

Page 2 of 3

patients, a total of 314 hospitalizations occurred within the study period (Figure 1). 52 men and 60 women were in this study, with a median age of 31 years (19-58 years) had a median 2.87 hospitalizations. In all these hospitalizations reviewed, 276 PICC line were inserted and 38 Port-a-Caths were either inserted or present. $87.3 \%$ of the catheters

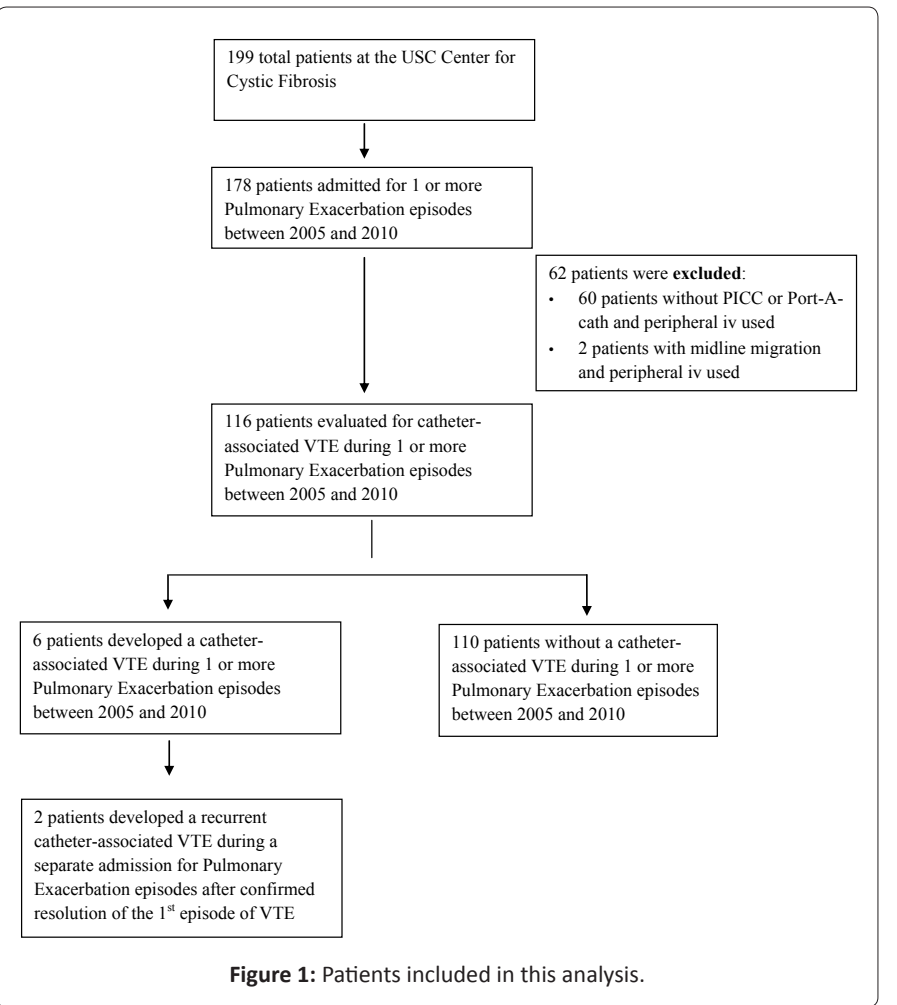

were inserted by the specialized nurse and $12.7 \%$ by the specialized radiologist.

Six of the 116 patients developed symptomatic catheter-associated VTE. Two patients (both with the Port-a-Cath) developed a recurrent VTE during a separate admission. This total of 8 thrombotic events proven by duplex ultrasound gives an incidence of $2.5 \%$. Age did not appear to be a factor in developing catheter-associated VTE $(30 \pm 4.8$ years VTE versus $32 \pm 8$.5years no VTE). Table1 shows the differences among the groups with and without associated VTE. Risk factors such as contraceptive use, associated malignancy or nephropathy and smoking did not reveal an impact on the development of a VTE. A higher CRP was associated with the thrombosis group, but did not reach statistical significance (p-value 0.51 ). None of 6 patients with confirmed catheterassociated VTE had Burkholderia cepacia respiratory infection.

The time to a catheter- associated VTE was shorter with the subset of patients who received a PICC compared to a Port-a-Cath (Table 2). There were only two patients with recurrent VTE. Both these patients had a Port-a-Cath during the admission and both developed nonmassive pulmonary embolus. There were no recurrent VTE in the patients with PICC line placement.

\section{Discussion}

The development of a venous thrombus is multifactorial in nature. Common acquired and inherited risk factors associated with VTE development in the cystic fibrosis population include inflammatory illness that are associated with infections (particularly Burkholderia cepacia), immobility issues, surgery, thrombophilia (altered protein $\mathrm{C}$, protein S, lupus anticoagulant and antithrombin III level or function) and platelet aggregation [2-6]. In these mentioned studies, genotype did not account for a contributing factor, nor was there a gender predominance to develop a catheter associated VTE. Our goal was not

\begin{tabular}{|c|c|c|}
\hline 314 total admissions & VTE cases $(n=8)$ & Controls $(n=306)$ \\
\hline Total Admissions, gender (Female/Male) & $8(7 / 1)$ & $304(161 / 145)$ \\
\hline Age (mean $\pm S D$ ), in years & $29.87 \pm 4.76$ & $32.02 \pm 8.45$ \\
\hline \multicolumn{3}{|c|}{ Contraceptive use in women (total number of women $=60$ ) } \\
\hline None & $85 \%$ (6 women) & $74 \%$ (39 women) \\
\hline Ever & $15 \%$ (1 woman) & $26 \%$ (14 women) \\
\hline \multicolumn{3}{|l|}{ Nephropathy } \\
\hline None & $100 \%$ (8 patients) & 93\% (101 patients) \\
\hline Hypertensive & 0 & $2 \%$ (2 patients) \\
\hline Diabetic & 0 & $5 \%$ (5 patients) \\
\hline \multicolumn{3}{|l|}{ Smoker (total number of patients $=116$ ) } \\
\hline Never & $100 \%$ (8 patients) & 99\% (286 patients) \\
\hline Ever & 0 & $1 \%$ (2 patients) \\
\hline $\mathrm{CRP}($ mean $\pm \mathrm{SD}), \mathrm{mg} / \mathrm{L}$ & $27.87 \pm 32.25$ & $19.14 \pm 34.57$ \\
\hline
\end{tabular}

Table 1: Baseline characteristics between the two cohorts.

\begin{tabular}{|c|c|c|c|c|}
\hline Patient No. & Type of Catheter & Days to VTE & Treatment Type \& Duration & Recurrence; Time to $2^{\text {nd }}$ Event \\
\hline 1 & PICC days & 3 & Warfarin, 3 months & No \\
\hline 2 & PICC days & 17 & Warfarin, 4 months & No \\
\hline 3 & PICC days & 21 & Warfarin, 3.5 months & No \\
\hline 4 & PICC days & 9 & Warfarin, 3 months & Yes; 582 days \\
\hline 5 & Port-A-cath & 4380 days & $\begin{array}{c}\text { iv heparin, thrombolytics } \\
\text { fays, then Warfarin } \\
\text { for } 6 \text { months }\end{array}$ & $\begin{array}{c}\text { iv heparin, thrombolytics } \\
\text { for 3 days, then Warfarin } \\
\text { for } 6 \text { months }\end{array}$ \\
\hline 6 & Port-A-cath & Y050 days & Yes; 912 days \\
\hline
\end{tabular}

Table 2: Details of CF Patients in Whom Catheter-Associated VTE Occurred. 
to replicate existing analysis, but most of the incidence reports in the CF population are based on the pediatric population.

Of the adult CF population the majority of the catheter-associated VTE's have been reported in patients with an indwelling catheter, such a Port-A-cath. In a study by Yung et al., three episodes of a VTE occurred related to 33 Port-a-Cath insertions over an 8 year period for adult patients with CF [7]. The time to VTE development ranged between 99 and 666 days. Similarly 4 out of 61 tunneled catheters inserted in adult patients with CF was reported by Rodgers et al. [8]. There was no mention of time to VTE development. More recently, Aitken et al. reviewed their cohort of adult CF patients with an indwelling catheter over a 10-year period [9]. 14 out of 61 patients with an indwelling catheter developed a thrombus (incidence 23\%). Two patients developed a subsequent pulmonary embolus. Eight patients were evaluated for a hypercoaguable state and all had normal clotting factors.

There has been only 1 other trial evaluating the VTE development with insertion of a PICC line with an incidence of $8.2 \%$. During a pulmonary exacerbation, CF patients generally have a higher systemic inflammatory state. Nash et al. reported serum ESR before PICC line insertion to be higher (mean $54.4 \mathrm{~mm} / \mathrm{hr}$ ) in those developing a venous thrombus compared to those without a PICC line (mean ESR $38.4 \mathrm{~mm} /$ hr) [10].

In this single institution study, we tasked ourselves to increase the understanding of the discrepancy for adult CF patients to develop catheter associated thromboses. Potential thrombosis associated risk factors were compared to the general population including smoking, history of contraceptive use and the presence of malignancy or nephropathy $[11,12]$. These risk factors did not play a pivotal role in the development of catheter-associated thrombosis in our analysis. Although there was a slight trend of increased CRP levels in our analysis with the thrombosis group, it did not meet statistical significance.

In this study of 116 patients with cystic fibrosis over a 5-year period, there were a total of 314 hospital admissions for pulmonary exacerbation. This accounted for an incidence of catheter-associated thrombosis of $2.5 \%$, which is a rate lower than $4.7 \%$ reported by Munck and Nash $[10,13]$. This incidence is also lower than expected in the general popular at $3 \%$ incidence [14]. It should be noted that the incidence we are reporting are only those diagnosed in cystic fibrosis patients requiring intravenous antibiotics for a prolonged period of time. All 8 cases of thrombosis were given anticoagulation with Coumadin for a 3 to 6 -month in order to prevent recurrence. Although INR therapeutic range was kept at the lower target range (1.8-2.3) due to the particular concern for massive hemoptysis, there was no observed worsening of the thrombus burden [15]. Only one of the patients on anticoagulation had a hemoptysis episode, but the quantity was small and did not warrant any discontinuation of the anticoagulation therapy.

As with all retrospective studies, this study is not without limitations. At the Keck Hospital of USC, electronic medical charting has not been fully instituted. The collections and review of the data collection from 2005 through 2010 were paper charting. Some medical information was missing and thus excluded, thereby reducing the number of patients allowable for evaluation. Also, routine surveillance for asymptomatic venous thrombosis has been routinely performed at our institution.

The results of this study provide clinicians with an estimate of catheter-associated thrombotic complications. Overall implantable vascular access devices are relatively safe and reliable for long term and frequent intravenous antibiotic therapy in patients with cystic fibrosis. Larger prospective studies are required to better delineate the risks for catheter-associated thrombosis in patients with cystic fibrosis.

\section{References}

1. Flume PA, Mogayzel PJ Jr, Robinson KA, Goss CH, Rosenblatt RL, et al. (2009) Cystic fibrosis pulmonary guidelines: treatment of pulmonary exacerbations. Am J Respir Crit Care Med 180: 802-808.

2. Balfour-Lynn IM, Malbon K, Burman JF, Davidson SJ (2005) Thrombophilia in children with cystic fibrosis. Pediatr Pulmonol 39: 306-310.

3. Raffini LJ, Raybagkar D, Blumenstein MS, Rubenstein RC, Manno CS (2006) Cystic fibrosis as a risk factor for recurrent venous thrombosis at a pediatric tertiary care hospital. J Pediatr 148: 659-664.

4. Barker M, Thoenes D, Döhmen H, Friedrichs F, Pfannenstiel C, et al. (2005) Prevalence of thrombophilia and catheter-related thrombosis in cystic fibrosis. Pediatr Pulmonol 39: 156-161.

5. Williams V, Griffiths AB, Yap ZL, Martin J, Smith G, et al. (2010) Increased thrombophilic tendency in pediatric cystic fibrosis patients. Clin Appl Thromb Hemost 16: 71-76.

6. O'Sullivan BP, Linden MD, Frelinger AL 3rd, Barnard MR, Spencer-Manzon M et al. (2005) Platelet activation in cystic fibrosis. Blood 105: 4635-4641.

7. Yung B, Campbell IA, Elborn JS, Harvey JS, Shale DJ (1996) Totally implantable venous access devices in adult patietns with cystic fibrosis. Respir Med 90:353 356

8. Rodgers HC, Liddle K, Nixon SJ, Innes JA, Greening AP (1998) Totally implantable venous access devices in cystic fibrosis and patient's opinion. Eur Respir J 1998;12: 217-220

9. Aitken ML, Tonelli MR (2000) Complications of indwelling catheters in cystic fibrosis: a 10-year review. Chest 118: 1598-1602.

10. Nash EF, Helm EJ, Stephenson A, Tullis E (2009) Incidence of deep vein thrombosis associated with peripherally inserted central catheters in adults with cystic fibrosis. J Vasc Interv Radiol 20: 347-351.

11. Heit JA, Silverstein MD, Mohr DN, Petterson TM, O'Fallon WM, et al. (2000) Risk factors for deep vein thrombosis and pulmonary embolism: a populationbased case-control study. Arch Intern Med 160: 809-815.

12. Sagar S, Stamatakis JD, Thomas DP, Kakkar VV (1976) Oral contraceptives antithrombin- III activity, and postoperative deep-vein thrombosis. Lancet 1 509-511.

13. Munck A, Malbezin S, Bloch J, Gerardin M, Lebourgeois M, et al. (2004) Follow up of 452 totally implantable vascular devices in cystic fibrosis patients .Eur Respir J 23: 430-434.

14. Evans RS, Sharp JH, Linford LH, Lloyd JF, Tripp JS, et al. (2010) Risk of symptomatic DVT associated with peripherally inserted central catheters. Chest 138: 803-810.

15. Ageno W, Gallus AS, Wittkowsky A (2012) Antithrombotic therapy and prevention of thrombosis, 9th ed: American College of Chest Physicians evidence-based clinical practice guidelines. CHEST 141(Suppl): e44S-e88s. 